\title{
Virtual actuator-based FTC for LPV systems with saturating actuators and FDI delays
}

\author{
Damiano Rotondo, Jean-Christophe Ponsart, Fatiha Nejjari, Didier Theilliol and Vicenç Puig
}

\begin{abstract}
The main contribution of this paper consists in solving the problem of fault tolerant control (FTC) for linear parameter varying (LPV) systems subject to actuator saturation and fault detection and isolation (FDI) delays. The FTC is based on virtual actuators that reconfigure the faulty plant to maintain the stability and to avoid the saturation of the actuators. On the other hand, a design methodology that provides the nominal output-feedback controller, which maximizes the tolerated delay between the fault occurrence and its isolation, is developed. The design process consists in finding the optimal feasible solution to a finite set of linear matrix inequalities (LMIs). Finally, an example is used to illustrate the theoretical results.
\end{abstract}

\section{INTRODUCTION}

Control systems are affected by saturations, which means that the control action that can be delivered by the actuators is limited in magnitude [1]. It is important to take into account the presence of saturations during the design of a control system, otherwise its real performance could exhibit strong performance degradation and, in some cases, instability could occur as well. For this reason, the problem of stabilizing systems with saturated inputs is extensively studied, as demonstrated by a number of works that have appeared recently in the literature, see e.g. [2]-[5]. Several control techniques have been extended to cope with input saturations, such as sliding mode control [6] and adaptive control [7]. Also, a few monographies have been published about this topic, see e.g. [8] and [9].

The presence of actuator saturations becomes even more problematic when actuator faults are considered. In the last decades, fault tolerant control (FTC) techniques have been investigated [10]-[12] with the objective of mitigating

This work has been funded by the Spanish Government (MINECO) through the project CICYT ECOCIS (ref. DPI2013-48243-C2-1-R), by MINECO and FEDER through the project CICYT HARCRICS (ref. DPI2014-58104-R), by AGAUR through the contract FI-DGR 2015 (ref. 2015FI_B2 00171) and by the DGR of Generalitat de Catalunya (SAC group Ref. 2014/SGR/374). D. Rotondo also acknowledges that part of this work was carried out during the tenure of an ERCIM Alain Bensoussan Fellowship Programme and that it was partly supported by the Research Council of Norway through the Centres of Excellence funding scheme, Project number 223254.

D. Rotondo, F. Nejjari and V. Puig are with Advanced Control Systems Group (SAC), Universitat Politècnica de Catalunya (UPC), Rambla de Sant Nebridi, 11, 08222 Terrassa, Spain. D. Rotondo is also with the Centre for Autonomous Marine Operations and Systems (NTNUAMOS), Department of Engineering Cybernetics, Norwegian University of Science and Technology, Trondheim, Norway. V. Puig is also with the Institut de Robòtica i Informàtica Industrial (IRI), UPC-CSIC, Carrer de Llorens i Artigas, 4-6, 08028 Barcelona, Spain. J.-C. Ponsart and D. Theilliol are with Université de Lorraine, CRAN, UMR 7039, Campus Sciences, B.P. 70239, Vandoeuvre-les-Nancy Cedex 54506 and with CNRS, CRAN, UMR 7039. Corresponding author: Damiano Rotondo (e-mail: damiano.rotondodyahoo.it) the effects of faults on the closed-loop performance and stability. These techniques usually lead to a redistribution of the control action among the healthy actuators, which can potentially become saturated. In order to avoid this potential actuator saturation, active FTC systems that include a trajectory replanning algorithm have been proposed [13][15].

Recently, an FTC strategy for unstable linear time invariant (LTI) systems subject to actuator saturation and fault isolation delay has been developed [16]. The proposed strategy relied on virtual actuators, a fault-hiding technique that aims at reconfiguring the faulty plant instead of the controller [17]-[21]. In [22], this strategy has been extended to linear parameter varying (LPV) systems, a standard formalism for performing gain scheduling of nonlinear systems [23]. However, in [22], a perfect fault detection and isolation (FDI) has been assumed. In some cases, the time delay between the appearance of the fault and the moment in which the active strategy is activated (at the fault detection or isolation time) may destabilize the system. The main contribution of the present work is to include a maximization of the tolerated FDI delay within the design process, which is done by means of a linear matrix inequality (LMI)-based design.

The paper is structured as follows. Section II recalls some preliminary results. Section III describes the system, the faults and the nominal controller. The virtual actuator-based FTC strategy is presented in Section IV. The design of the nominal controller through maximization of the tolerated FDI delay is described in Section V. Section VI illustrates the theoretical results through an example. Finally, Section VII summarizes the main conclusions.

\section{PRELIMINARIES}

Consider the autonomous nonlinear system:

$$
\dot{x}(t)=g(x(t))
$$

where $x \in \mathbb{R}^{n_{x}}$ is the state and $g$ denotes a nonlinear function. For $x(0)=x_{0} \in \mathbb{R}^{n_{x}}$, let us denote the trajectory of the system (1) as $\psi\left(t, x_{0}\right)$. Then, the domain of attraction of the origin is:

$$
S:=\left\{x_{0} \in \mathbb{R}^{n_{x}}: \lim _{t \rightarrow+\infty} \psi\left(t, x_{0}\right)=0\right\}
$$

Let $P \succ 0, \rho>0$ and denote:

$$
\mathcal{E}(P, \rho)=\left\{x_{0} \in \mathbb{R}^{n_{x}}: x^{\mathrm{T}} P x \leq \rho\right\}
$$

and let $V(x(t))=x(t)^{\mathrm{T}} P x(t)$ be a candidate Lyapunov function. The ellipsoid $\mathcal{E}(P, \rho)$ is said to be contractively invariant if $\dot{V}(x(t))<0$ for all $x \in \mathcal{E}(P, \rho) \backslash\{0\}$. A contractively 
invariant ellipsoid $\mathcal{E}(P, \rho)$ is inside the domain of attraction $\mathcal{S}$ [24].

Finally, throughout the paper, $H e\{M\}$ will be used as a shorthand notation for the matrix $M+M^{T}$.

\section{SySTEM, FAULT AND CONTROLLER DEFINITION}

Let us consider the following LPV system subject to actuator saturations:

$$
\begin{aligned}
\dot{x}(t) & =A(\theta(t)) x(t)+\mathfrak{B}(t) s a t(u(t)) \\
y(t) & =C x(t)
\end{aligned}
$$

where $x \in \mathbb{R}^{n_{x}}$ is the state, $u \in \mathbb{R}^{n_{u}}$ is the control input, $y \in \mathbb{R}^{n_{y}}$ is the measured output, $A(\theta(t)) \in \mathbb{R}^{n_{x} \times n_{x}}$ is the parameter varying state matrix, whose values depend on the vector $\theta(t) \in \Theta \subset \mathbb{R}^{n_{\theta}}, C \in \mathbb{R}^{n_{y} \times n_{x}}$ is the output matrix, and $\mathfrak{B}(t) \in \mathbb{R}^{n_{x} \times n_{u}}$ is the input matrix, defined as follows:

$$
\mathfrak{B}(t)= \begin{cases}B & t<t_{f} \\ B_{f} \in\left\{B_{f}^{(1)}, \ldots, B_{f}^{\left(n_{f}\right)}\right\} & t \geq t_{f}\end{cases}
$$

$B \in \mathbb{R}^{n_{x} \times n_{u}}$ and the corresponding LPV system obtained from (4)-(5) will be referred to as nominal input matrix and nominal system, respectively, $B_{f} \in \mathbb{R}^{n_{x} \times n_{u}}$ and the corresponding LPV system obtained from (4)-(5) will be referred to as faulty input matrix and faulty system, respectively, and $t_{f} \in \mathbb{R}^{+}$is the fault occurrence time.

Remark 1: The fault definition provided by (6) is a general formulation that may specify both faults in a single actuator and in multiple actuators. For example, $B_{f}^{(1)}$ could represent the total loss of actuator 1 , while $B_{f}^{(2)}$ could represent the total loss of actuators 1 and 2 .

The saturation function sat $: \mathbb{R}^{n_{u}} \rightarrow \mathbb{R}^{n_{u}}$ specifies the limited actuator capacity of the control input $u(t)$ in (4). The saturation is assumed to be a symmetrical, decoupled, sectorbounded and static actuator nonlinearity with a constant saturation limit $u_{j}^{M A X}$ in the $j$-th input, such that:

$$
\begin{gathered}
\operatorname{sat}(u)=\left(\begin{array}{c}
\operatorname{sat}_{1}\left(u_{1}\right) \\
\vdots \\
\operatorname{sat}_{j}\left(u_{j}\right) \\
\vdots \\
\operatorname{sat}_{n_{u}}\left(u_{n_{u}}\right)
\end{array}\right) \\
\operatorname{sat}_{j}\left(u_{j}\right)=\left\{\begin{array}{cc}
u_{j}^{M A X} & \text { if } u_{j}>u_{j}^{M A X} \\
u_{j} & \text { if }\left|u_{j}\right| \leq u_{j}^{M A X} \\
-u_{j}^{M A X} & \text { if } u_{j}<-u_{j}^{M A X}
\end{array}\right.
\end{gathered}
$$

for $j=1, \ldots, n_{u}$, where $u^{M A X}=\left(u_{1}^{M A X}, \ldots, u_{n_{u}}^{M A X}\right)^{\mathrm{T}} \in \mathbb{R}^{n_{u}}$ is a vector with positive entries.

The $n_{f}$ matrices $B_{f}^{(1)}, \ldots, B_{f}^{\left(n_{f}\right)} \in \mathbb{R}^{n_{x} \times n_{u}}$ are such that:

$$
\operatorname{rank}\left(B_{f}^{(h)}\right)<\operatorname{rank}(B)
$$

and the pairs:

$$
\left(A(\theta), B_{f}^{(h)}\left(B_{f}^{(h)}\right)^{\dagger} B\right)
$$

are stabilizable, $\forall \theta \in \Theta$ and $\forall h=1, \ldots, n_{f}$.

The structure chosen for the controller is the LPV dynamic feedback one [25]:

$$
\begin{aligned}
& \dot{x}_{c}(t)=A_{c}(\theta(t)) x_{c}(t)+B_{c}(\theta(t)) y(t) \\
& u_{c}(t)=C_{c}(\theta(t)) x_{c}(t)+D_{c}(\theta(t)) y(t)
\end{aligned}
$$

where $x_{c} \in \mathbb{R}^{n_{x}}$ is the controller state and $u_{c} \in \mathbb{R}^{n_{u}}$ is the controller output, respectively. A strict requirement for the closed-loop system obtained by considering the system (4)(5) and the controller (11)-(12) is that $\mathcal{E}(P, 1) \subseteq \mathcal{S}$ and $\mathcal{E}(P, 1) \subseteq \mathcal{L}\left(u, u^{M A X}\right)$, where $\mathcal{L}\left(u, u^{M A X}\right)$ denotes the region of the state space in which the actuators are not saturated.

In other words, the controller must be such that for any initial closed-loop state vector satisfying:

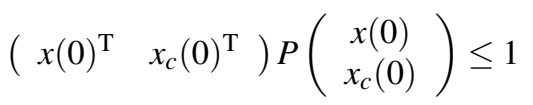

the control input never saturates, and the closed-loop state trajectory converges to the origin.

\section{ViRTUAL ACTUATOR-BASED FTC}

The FTC strategy used in this work relies on a reconfigured control law, given by:

$$
u(t)=\left\{\begin{array}{cc}
u_{c}(t) & t<t_{I} \\
u_{f}^{(1)}(t) & t \geq t_{I}, \mathfrak{B}(t)=B_{f}^{(1)} \\
\vdots & \vdots \\
u_{f}^{\left(n_{f}\right)}(t) & t \geq t_{I}, \mathfrak{B}(t)=B_{f}^{\left(n_{f}\right)}
\end{array}\right.
$$

where $t_{I} \in \mathbb{R}^{+}, t_{I} \geq t_{f}$ is the fault isolation time, that is assumed to be provided by an FDI module. The values for $u_{f}^{(1)}(t), \ldots, u_{f}^{\left(n_{f}\right)}(t)$ are provided by the LPV virtual actuators, which have a structure as in [22]:

$$
\begin{aligned}
& \dot{x}_{v}^{(h)}(t)=\left(A(\theta(t))+B_{*}^{(h)} M^{(h)}(\theta(t))\right) x_{v}^{(h)}(t)+\left(B-B_{*}^{(h)}\right) u_{c}(t) \\
& u_{f}^{(h)}(t)=N^{(h)}\left(u_{c}(t)-M^{(h)}(\theta(t)) x_{v}^{(h)}(t)\right)
\end{aligned}
$$

where $h=1, \ldots, n_{f}, x_{v}^{(h)}$ are the virtual actuators states with $x_{v}^{(h)}\left(t_{I}\right)=0, M^{(h)}(\theta(t)) \in \mathbb{R}^{n_{u} \times n_{x}}$ are the virtual actuators gains to be designed, and the matrices $N^{(h)}$ and $B_{*}^{(h)}$ are given by:

$$
\begin{aligned}
& N^{(h)}=\left(B_{f}^{(h)}\right)^{\dagger} B \\
& B_{*}^{(h)}=B_{f}^{(h)} N^{(h)}=B_{f}^{(h)}\left(B_{f}^{(h)}\right)^{\dagger} B
\end{aligned}
$$

Moreover, in order to obtain the fault-hiding characteristic, the output equation (5) is slightly changed after $t_{I}$, as follows:

$$
y(t)=C\left(x(t)+x_{v}^{(h)}(t)\right) \quad t \geq t_{I}, \mathfrak{B}(t)=B_{f}^{(h)}
$$

Given a controller (11)-(12), satisfying $\mathcal{E}(P, 1) \subseteq \mathcal{S}$ and $\mathcal{E}(P, 1) \subseteq \mathcal{L}\left(u, u^{M A X}\right)$, the following theorem provides the conditions to design the virtual actuator guaranteeing that, if at the fault isolation time $t_{I}$, the closed-loop system state is inside the ellipsoid $\mathcal{E}\left(P, v_{f}\right)$, with $\left.\left.v_{f} \in\right] 0,1\right]$, the state 
trajectory will converge to zero despite the change of the input matrix from $B$ to $B_{f}^{(h)}$ due to the fault.

Theorem 1: Let $X_{v a}^{-1} \in \mathbb{S}^{n_{x} \times n_{x}}$ and $\Gamma^{(h)}(\theta) \in \mathbb{R}^{n_{u} \times n_{x}}, h=$ $1, \ldots, n_{f}$ be such that:

$$
\begin{aligned}
& H e\left\{\left(\begin{array}{cc}
v_{f} A_{c l}(\theta) P^{-1} & 0 \\
v_{f} A_{*}^{(h)}(\theta) P^{-1} & A(\theta) X_{v a}^{-1}+B_{*}^{(h)} \Gamma^{(h)}(\theta)
\end{array}\right)\right\} \prec 0
\end{aligned}
$$

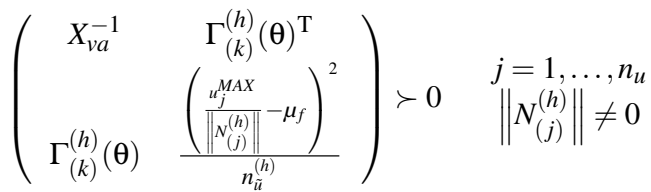

hold $\forall \theta \in \Theta$, where:

$$
\begin{aligned}
& A_{c l}(\theta)=\left(\begin{array}{cc}
A(\theta)+B D_{c}(\theta) C & B C_{c}(\theta) \\
B_{c}(\theta) C & A_{c}(\theta)
\end{array}\right)
\end{aligned}
$$

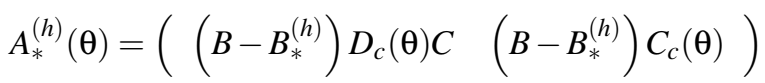

$$
\begin{aligned}
& \mu_{f}=\max _{\mathcal{E}\left(P, v_{f}\right)}\left\|u_{c}\right\|
\end{aligned}
$$

$n_{\tilde{u}}^{(h)}$ is the number of non-zero elements in $N_{(j)}^{(h)}$, and $k$ in (21) takes values corresponding to the indices of the nonzero elements in $N_{(j)}^{(h)}$. Then, if the virtual actuators gains $M^{(h)}(\theta)$ in (15)-(16) are calculated as $M^{(h)}(\theta)=\Gamma^{(h)}(\theta) X_{v a}$, $\mathcal{E}\left(P, \vee_{f}\right)$ is contractively invariant for the system (4)-(5) with the control law (14), and $\mathcal{E}\left(P, v_{f}\right) \subseteq \mathcal{L}\left(u, u^{M A X}\right), \forall t \geq t_{I}$.

Proof: See [22].

By relying on a polytopic representation, it is possible to transform the infinite number of conditions provided by Theorem 1 in a finite number of conditions, as stated by the following corollary.

Corollary 1: Let the matrices $A(\theta(t)), A_{c}(\theta(t)), B_{c}(\theta(t))$, $C_{c}(\theta(t)), D_{c}(\theta(t))$ be polytopic, i.e.:

$$
\left(\begin{array}{c}
A(\theta(t)) \\
A_{c}(\theta(t)) \\
B_{c}(\theta(t)) \\
C_{c}(\theta(t)) \\
D_{c}(\theta(t))
\end{array}\right)=\sum_{i=1}^{N} \mu_{i}(\theta(t))\left(\begin{array}{c}
A_{i} \\
A_{c, i} \\
B_{c, i} \\
C_{c, i} \\
D_{c, i}
\end{array}\right)
$$

with coefficients $\mu_{i}(\theta(t))$ such that:

$$
\sum_{i=1}^{N} \mu_{i}(\theta(t))=1, \mu_{i}(\theta(t)) \geq 0, \forall i=1, \ldots, N, \forall \theta \in \Theta
$$

and choose the virtual actuator gain as:

$$
M^{(h)}(\theta(t))=\sum_{i=1}^{N} \mu_{i}(\theta(t)) M_{i}^{(h)}
$$

If there exist $X_{v a}^{-1} \in \mathbb{S}^{n_{x} \times n_{x}}$ and $\Gamma_{i}^{(h)} \in \mathbb{R}^{n_{u} \times n_{x}}, h=1, \ldots, n_{f}$, $i=1, \ldots, N$ such that:

$$
H e\left\{\left(\begin{array}{cc}
v_{f} A_{c l, i} P^{-1} & O_{2 n_{x} \times n_{x}} \\
v_{f} A_{*, i}^{(h)} P^{-1} & A_{i} X_{v a}^{-1}+B_{*}^{(h)} \Gamma_{i}^{(h)}
\end{array}\right)\right\} \prec O
$$

$$
\left(\begin{array}{cc}
X_{v a}^{-1} & \left(\Gamma_{i(k)}^{(h)}\right)^{\mathrm{T}} \\
\Gamma_{i(k)}^{(h)} & \frac{\left(\begin{array}{l}
u_{j}^{M A X} \\
\left\|N_{j}^{(h)}\right\|^{-}
\end{array}\right)^{2}}{n_{\tilde{u}}^{(h)}}
\end{array}\right) \succ O \quad \begin{aligned}
& j=1, \ldots, n_{u} \\
& \left\|N_{j}^{(h)}\right\| \neq 0
\end{aligned}
$$

hold $\forall i=1, \ldots, N$, where:

$$
\begin{aligned}
& A_{c l, i}=\left(\begin{array}{cc}
A_{i}+B D_{c, i} C & B C_{c, i} \\
B_{c, i} C & A_{c, i}
\end{array}\right)
\end{aligned}
$$

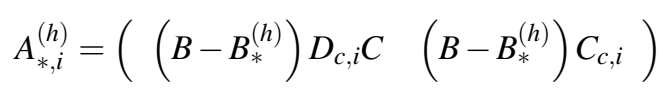

$\mu_{f}$ is defined as in (24), $n_{\tilde{u}}^{(h)}$ is the number of non-zero elements in $N_{(j)}^{(h)}$, and $k$ in (29) takes values corresponding to the indices of the non-zero elements in $N_{(j)}^{(h)}$, and if the vertex virtual actuators gains in (27) are calculated as $M_{i}^{(h)}=\Gamma_{i}^{(h)} X_{v a}$, then $\mathcal{E}\left(P, v_{f}\right)$ is contractively invariant for the system (4)-(5) with control law (14), and $\mathcal{E}\left(P, \vee_{f}\right) \subseteq$ $\mathcal{L}\left(u, u^{M A X}\right), \forall t \geq t_{I}$.

Proof: See [22].

\section{DESIGN OF THE CONTROLLER}

Under the assumption of instantaneous fault isolation, i.e. $t_{I}=t_{f}$, if the output-feedback controller has been designed such that $\mathcal{E}(P, 1) \subseteq \mathcal{S}$ and $\mathcal{E}(P, 1) \subseteq \mathcal{L}\left(u, u^{M A X}\right)$ and if the closed-loop state trajectory has reached $\mathcal{E}\left(P, v_{f}\right)$, the virtual actuator-based FTC described in Section IV guarantees the state trajectory convergence under fault occurrence. However, this is not the case when there is a delay in the fault isolation, i.e. $t_{I}-t_{f}>0$. In fact, between the occurrence of the fault, that changes the system input matrix from $B$ to some $B_{f}^{(h)}$, and the fault isolation time, when the appropriate control $u_{f}^{(h)}$ begins to be applied, there is a time interval where the system is driven by the nominal control $u_{c}(t)$. During this period, there is no guarantee that, if the system has reached $\mathcal{E}\left(P, v_{f}\right)$ at time $t_{f}$, it will stay inside this region until $t_{I}$. This fact can lead to severe consequences, because if the state trajectory leaves $\mathcal{E}\left(P, v_{f}\right)$ before $t_{I}$, the system could be destabilized [26]. Hence, it is interesting to improve the overall system robustness against the fault isolation delay.

Given $\left[\begin{array}{ll}x\left(t_{f}\right)^{\mathrm{T}} & x_{c}\left(t_{f}\right)^{\mathrm{T}}\end{array}\right]^{\mathrm{T}} \in \mathcal{E}\left(P, v_{f}\right)$, let us define, for the faulty system (4)-(5) with $\mathfrak{B}(t)=B_{f}^{(h)}, h=1, \ldots, n_{f}$, under control law $u(t)=u_{c}(t)$, the critical fault isolation time $\widehat{t}_{I}^{(h)}\left(x\left(t_{f}\right), x_{c}\left(t_{f}\right)\right) \geq t_{f}$ as the time instant such that:

$$
\left[x\left(\widehat{t}_{I}^{(h)}\right)^{T} x_{c}\left(\widehat{t}_{I}^{(h)}\right)^{T}\right]^{T} \in \mathcal{E}\left(P, v_{f}\right)
$$

but:

$$
\left[x \left(\left(_{t}^{(h)}+t_{\varepsilon}\right)^{T} \quad x_{c}\left(\left(_{I}^{(h)}+t_{\varepsilon}\right)^{T}\right]^{T} \notin \mathcal{E}\left(P, v_{f}\right)\right.\right.
$$

for all $t_{\varepsilon}>0$. The critical fault isolation time indicates that the guarantees of non-saturating control input and state trajectory convergence to the origin given by the virtual actuator-based FTC are lost if $t_{I}>\widehat{t}_{I}^{(h)}\left(x\left(t_{f}\right), x_{c}\left(t_{f}\right)\right)$. It is 
worth remarking that the Lyapunov-based conditions provided in the following are sufficient, such that the system may exhibit state trajectory convergence to zero with nonsaturating control input even if $t_{I}>\widehat{t}_{I}^{(h)}\left(x\left(t_{f}\right), x_{c}\left(t_{f}\right)\right)$.

In the following, the output feedback controller (11)(12) will be designed in such a way that it maximizes $\min _{h=1, \ldots, n_{f}} \hat{t}_{I}^{(h)}\left(x\left(t_{f}\right), x_{c}\left(t_{f}\right)\right)$ for all $\left[\begin{array}{ll}x\left(t_{f}\right)^{\mathrm{T}} & x_{c}\left(t_{f}\right)^{\mathrm{T}}\end{array}\right]^{\mathrm{T}} \in$ $\mathcal{E}\left(P, v_{f}\right)$, where $\hat{t}_{I}^{(h)}\left(x\left(t_{f}\right), x_{c}\left(t_{f}\right)\right)$ is an estimation of $\widehat{t}_{I}^{(h)}\left(x\left(t_{f}\right), x_{c}\left(t_{f}\right)\right)$.

As a first step to obtain the conditions for designing the output feedback controller (11)-(12), let us consider the following lemma.

Lemma 1: Let $V(x(t))=x(t)^{T} P x(t)$, and let $\mathcal{D}$ be an LMI region [27], i.e. a subset of the complex plane such that:

$$
\mathcal{D}=\left\{\sigma \in \mathbb{C}: f_{\mathcal{D}}(\sigma) \prec 0\right\}
$$

where $f_{\mathcal{D}}(\sigma)$ is the characteristic function defined as:

$$
f_{\mathcal{D}}(\sigma)=\alpha+\beta \sigma+\beta^{\mathrm{T}} \bar{\sigma}
$$

where $\alpha \in \mathbb{S}^{m \times m}, \beta \in \mathbb{R}^{m \times m}$, and $\bar{\sigma}$ denotes the complex conjugate of $\sigma$.

If the autonomous LPV system:

$$
\dot{x}(t)=A(\theta(t)) x(t)
$$

is quadratically $\mathcal{D}$ stable [27], i.e. there exists $P \succ 0$ such that $\forall \boldsymbol{\theta} \in \Theta$ :

$$
\alpha \otimes P+\beta \otimes P A(\theta)+\beta^{\mathrm{T}} \otimes A(\theta)^{\mathrm{T}} P \prec 0
$$

then, $V(x(t))$ satisfies, for all $x(t) \neq 0$ :

$$
\frac{1}{2} \frac{\dot{V}(x(t))}{V(x(t))} \in \mathcal{D} \cap \mathbb{R}
$$

Proof: Pre-multiplying (37) by $I \otimes x(t)^{\mathrm{T}}$, and postmultiplying it by $I \otimes x(t)$, respectively, the following is obtained for all $x(t) \neq 0$ :

$$
\alpha \otimes x(t)^{\mathrm{T}} P x(t)+\beta \otimes x(t)^{\mathrm{T}} P A(\theta(t)) x(t)+\beta^{\mathrm{T}} \otimes x(t)^{\mathrm{T}} A(\theta(t))^{\mathrm{T}} P x(t) \prec 0
$$

Recalling that:

$$
\frac{1}{2} \dot{V}(x(t))=x(t)^{\mathrm{T}} P A(\theta(t)) x(t)=x(t)^{\mathrm{T}} A(\theta(t))^{\mathrm{T}} P x(t)
$$

and dividing (39) by $V(x(t))$, this process leads to:

$$
\alpha \otimes 1+\beta \otimes \frac{1}{2} \frac{\dot{V}(x(t))}{V(x(t))}+\beta^{\mathrm{T}} \otimes \frac{1}{2} \frac{\dot{V}(x(t))}{V(x(t))} \prec 0
$$

which implies (38).

Lemma 1 is used in the subsequent theorem to obtain an estimation of $\hat{t}_{I}^{(h)}\left(x\left(t_{f}\right), x_{c}\left(t_{f}\right)\right)$ for all $\left[x\left(t_{f}\right)^{\mathrm{T}} \quad x_{c}\left(t_{f}\right)^{\mathrm{T}}\right]^{\mathrm{T}} \in \mathcal{E}\left(P, v_{f}\right)$. $\Theta:$

Theorem 2: Let $\lambda^{(h)} \in \mathbb{R}^{+}, h=1, \ldots, n_{f}$, be such that $\forall \theta \in$

$-2 \lambda^{(h)} P+H e\left\{P\left(\begin{array}{cc}A(\theta)+B_{f}^{(h)} D_{c}(\theta) C & B_{f}^{(h)} C_{c}(\theta) \\ B_{c}(\theta) C & A_{c}(\theta)\end{array}\right)\right\} \prec 0$ and let $\left[\begin{array}{ll}x\left(t_{f}\right)^{\mathrm{T}} & x_{c}\left(t_{f}\right)^{\mathrm{T}}\end{array}\right]^{\mathrm{T}}=x_{f} \in \mathcal{E}\left(P, v_{f}\right)$. Then:

$$
\left[\begin{array}{ll}
x(t)^{\mathrm{T}} & x_{c}(t)^{\mathrm{T}}
\end{array}\right]^{\mathrm{T}} \in \mathcal{E}\left(P, v_{f}\right) \quad \forall t \in\left[t_{f}, \hat{t}_{I}^{(h)}\left(x\left(t_{f}\right), x_{c}\left(t_{f}\right)\right)\right]
$$

with:

$$
\hat{t}_{I}^{(h)}\left(x\left(t_{f}\right), x_{c}\left(t_{f}\right)\right)=t_{f}+\frac{1}{2 \lambda^{(h)}} \ln \left(\frac{\mathrm{v}_{f}}{x_{f}^{\mathrm{T}} P x_{f}}\right)
$$

Proof: The faulty system (4)-(5), with $\mathfrak{B}(t)=B_{f}^{(h)}$, together with the output feedback controller (11)-(12), can be rewritten in the closed-loop autonomous form as:

$$
\left(\begin{array}{c}
\dot{x}(t) \\
\dot{x}_{c}(t)
\end{array}\right)=\left(\begin{array}{cc}
A(\theta(t))+B_{f}^{(h)} D_{c}(\theta(t)) C & B_{f}^{(h)} C_{c}(\theta(t)) \\
B_{c}(\theta(t)) C & A_{c}(\theta(t))
\end{array}\right)\left(\begin{array}{c}
x(t) \\
x_{c}(t)
\end{array}\right)
$$

Let us apply Corollary 1 to (45) using the region $\operatorname{Re}(z)<$ $\lambda^{(h)}$, that corresponds to (35) with $\alpha=-2 \lambda^{(h)}$ and $\beta=1$, such that (37) reads as:

$-2 \lambda^{(h)} P+H e\left\{P\left(\begin{array}{cc}A(\theta)+B_{f}^{(h)} D_{c}(\theta) C & B_{f}^{(h)} C_{c}(\theta) \\ B_{c}(\theta) C & A_{c}(\theta)\end{array}\right)\right\} \prec 0$

Hence, if (46) holds, (38) is true for the quadratic function:

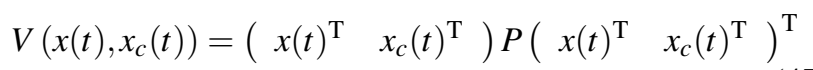

that implies:

$$
V\left(x(t), x_{c}(t)\right) \leq V\left(x_{f}\right) e^{2 \lambda^{(h)}\left(t-t_{f}\right)}=x_{f}^{\mathrm{T}} P x_{f} e^{2 \lambda^{(h)}\left(t-t_{f}\right)}
$$

By considering the condition $V\left(x(t), x_{c}(t)\right) \leq \mathrm{v}_{f}$, that defines $\mathcal{E}\left(P, \vee_{f}\right)$, it is straightforward to obtain (43).

From (44) it can be seen that, in order to maximize $\min _{h=1, \ldots, n_{f}} \hat{t}_{I}^{(h)}\left(x\left(t_{f}\right), x_{c}\left(t_{f}\right)\right)$, it is necessary to minimize $\lambda=\max _{h=1, \ldots, n_{f}} \lambda^{(h)}$. The solution to this problem is given by the following theorem.

Theorem 3: Let $X, Y \in \mathbb{S}^{n_{x} \times n_{x}}, F(\theta) \in \mathbb{R}^{n_{x} \times n_{y}}, K(\theta) \in$ $\mathbb{R}^{n_{u} \times n_{x}}, L(\theta) \in \mathbb{R}^{n_{u} \times n_{y}}, F^{(h)}(\theta) \in \mathbb{R}^{n_{x} \times n_{y}}$ and $N^{(h)}(\theta) \in$ $\mathbb{R}^{n_{x} \times n_{x}}, h=1, \ldots, n_{f}$, correspond to the solution to the following constrained minimization problem:

$$
\min \lambda
$$

subject to $\lambda \geq 0$ and:

$$
\begin{aligned}
& H e\{X A(\theta)+F(\theta) C\} \prec 0 \\
& H e\{A(\theta) Y+B K(\theta)\} \prec 0
\end{aligned}
$$

$$
\left(\begin{array}{ccc}
X & I & C L_{(j)}(\theta)^{\mathrm{T}} \\
I & Y & K_{(j)}(\theta)^{\mathrm{T}} \\
L_{(j)}(\theta) C & K_{(j)}(\theta) & \left(u_{j}^{M A X}\right)^{2}
\end{array}\right) \succ 0
$$

$-2 \lambda\left(\begin{array}{cc}X & I \\ I & Y\end{array}\right)+H e\left\{\left(\begin{array}{cc}X A(\theta)+F^{(h)}(\theta) C & N^{(h)}(\theta) \\ A(\theta)+B_{f}^{(h)} L(\theta) C & A(\theta) Y+B_{f}^{(h)} K(\theta)\end{array}\right)\right\} \prec 0$

$\forall j=1, \ldots, n_{u}, \forall h=1, \ldots, n_{f}$ and $\forall \theta \in \Theta$. 
Then, the output feedback controller (11)-(12), with matrices calculated as:

$$
\begin{aligned}
& \left(\begin{array}{cc}
A_{c}(\theta) & B_{c}(\theta) \\
C_{c}(\theta) & D_{c}(\theta)
\end{array}\right)=\left(\begin{array}{cc}
Z & X B \\
O & I
\end{array}\right)^{-1} \cdots \\
& \cdots\left(\begin{array}{cc}
-(A(\theta)+B L(\theta) C)^{\mathrm{T}}-X A(\theta) Y & F(\theta) \\
K(\theta) & L(\theta)
\end{array}\right)\left(\begin{array}{cc}
-Y & O \\
C Y & I
\end{array}\right)^{-1}
\end{aligned}
$$

$$
Z=X-Y^{-1}
$$

maximizes $\quad \min _{h=1, \ldots, n_{f}} \hat{t}_{I}^{(h)}\left(x\left(t_{f}\right), x_{c}\left(t_{f}\right)\right) \quad$ for $\quad$ all $\left[\begin{array}{ll}x\left(t_{f}\right)^{\mathrm{T}} & x_{c}\left(t_{f}\right)^{\mathrm{T}}\end{array}\right]^{\mathrm{T}} \in \mathcal{E}\left(P, v_{f}\right)$, where $\hat{t}_{I}^{(h)}\left(x\left(t_{f}\right), x_{c}\left(t_{f}\right)\right)$ is the estimation of $\hat{t}_{I}^{(h)}\left(x\left(t_{f}\right), x_{c}\left(t_{f}\right)\right)$ obtained as (44) with $P$ defined as:

$$
P=\left(\begin{array}{cc}
X & Z \\
Z & Z
\end{array}\right)
$$

Proof: If conditions (50)-(52) hold, then the closed-loop system obtained by considering the system (4)-(5) and the controller (11)-(12), with matrices calculated as in (54)-(55), is such that $\mathcal{E}(P, 1) \subseteq \mathcal{S}$ and $\mathcal{E}(P, 1) \subseteq \mathcal{L}\left(u, u^{M A X}\right)$, with $P$ defined as in (56).

On the other hand, the design condition (53) corresponds to the analysis condition (42) with $\lambda=\lambda^{(h)}$. In fact, by applying a congruent transformation to (42) with:

$$
\Gamma=\left(\begin{array}{cc}
I & 0 \\
Y & -Y
\end{array}\right)
$$

and $\lambda=\lambda^{(h)},(53)$ is obtained using the following change of variables:

$$
\begin{gathered}
N^{(h)}(\theta)=X A(\theta) Y-Z A_{c}(\theta) Y+Z B_{c}(\theta) C Y \\
-X B_{f}^{(h)} C_{c}(\theta) Y+X B_{f}^{(h)} D_{c}(\theta) C Y \\
F^{(h)}(\theta)=Z B_{c}(\theta)+X B_{f}^{(h)} D_{c}(\theta) \\
K(\theta)=-C_{c}(\theta) Y+D_{c}(\theta) C Y \\
L(\theta)=D_{c}(\theta)
\end{gathered}
$$

Since a common $\lambda$ is being used, it is clear that $\lambda=$ $\max _{h=1, \ldots, n_{f}} \lambda^{(h)}$, and by minimizing $\lambda$, we are maximizing $\min _{h=1, \ldots, n_{f}} \hat{t}_{I}^{(h)}$, defined as in (44).

Also in this case, by relying on a polytopic representation, it is possible to obtain conditions that can be applied for the controller design, as stated by the following corollary.

Corollary 2: Let the matrices $A(\theta(t)), A_{c}(\theta(t)), B_{c}(\theta(t))$, $C_{c}(\theta(t)), D_{c}(\theta(t))$ be polytopic, as in (25)-(26), and let $X, Y \in \mathbb{S}^{n_{x} \times n_{x}}, F_{i} \in \mathbb{R}^{n_{x} \times n_{y}}, K_{i} \in \mathbb{R}^{n_{u} \times n_{x}}, L_{i} \in \mathbb{R}^{n_{u} \times n_{y}}, F_{i}^{(h)} \in$ $\mathbb{R}^{n_{x} \times n_{y}}$ and $N_{i}^{(h)} \in \mathbb{R}^{n_{x} \times n_{x}}, h=1, \ldots, n_{f}, i=1, \ldots, N$, correspond to the solution of the constrained minimization problem (49):

$\min \lambda$

subject to $\lambda \geq 0$ and:

$$
\begin{aligned}
& H e\left\{X A_{i}+F_{i} C\right\} \prec 0 \\
& H e\left\{A_{i} Y+B K_{i}\right\} \prec 0
\end{aligned}
$$

$$
\begin{gathered}
\left(\begin{array}{ccc}
X & I & C L_{i(j)}^{\mathrm{T}} \\
I & Y & K_{i(j)}^{\mathrm{T}} \\
L_{i(j)} C & K_{i(j)} & \left(u_{j}^{M A X}\right)^{2}
\end{array}\right) \succ 0 \\
-2 \lambda\left(\begin{array}{cc}
X & I \\
I & Y
\end{array}\right)+H e\left\{\left(\begin{array}{cc}
X A_{i}+F_{i}^{(h)} C & N_{i}^{(h)} \\
A_{i}+B_{f}^{(h)} L_{i} C & A(\theta) Y+B_{f}^{(h)} K_{i}
\end{array}\right)\right\} \prec 0
\end{gathered}
$$

$\forall i=1, \ldots, N, \forall j=1, \ldots, n_{u}$ and $\forall h=1, \ldots, n_{f}$.

Then, the controller (11)-(12), with matrices calculated as:

$$
\begin{gathered}
\left(\begin{array}{cc}
A_{c, i} & B_{c, i} \\
C_{c, i} & D_{c, i}
\end{array}\right)=\left(\begin{array}{cc}
Z & X B \\
O & I
\end{array}\right)^{-1} \cdots \\
\cdots\left(\begin{array}{cc}
-\left(A_{i}+B L_{i} C\right)^{\mathrm{T}}-X A_{i} Y & F_{i} \\
K_{i} & L_{i}
\end{array}\right)\left(\begin{array}{cc}
-Y & O \\
C Y & I
\end{array}\right)^{-1}
\end{gathered}
$$

maximizes $\quad \min _{h=1, \ldots, n_{f}} \hat{t}_{I}^{(h)}\left(x\left(t_{f}\right), x_{c}\left(t_{f}\right)\right) \quad$ for $\quad$ all $\left[\begin{array}{ll}x\left(t_{f}\right)^{\mathrm{T}} & x_{c}\left(t_{f}\right)^{\mathrm{T}}\end{array}\right]^{\mathrm{T}} \in \mathcal{E}\left(P, v_{f}\right)$, where $\hat{t}_{I}^{(h)}\left(x\left(t_{f}\right), x_{c}\left(t_{f}\right)\right)$ is the estimation of $\hat{t}_{I}^{(h)}\left(x\left(t_{f}\right), x_{c}\left(t_{f}\right)\right)$ obtained as (44) with $P$ defined as in (56).

Proof: It follows from the basic property of matrices [28] that any linear combination of negative (positive) definite matrices with non-negative coefficients (whose sum is positive) is negative (positive) definite. Hence, using the coefficients $\mu_{i}(\theta(t))$, taking into account (26), (50)-(53) follow directly from (63)-(66).

\section{EXAMPLE}

Let us consider the open-loop unstable LPV system subject to actuator saturations proposed in [22], i.e. (4)-(5), with:

$$
\begin{aligned}
& A(\theta(t))=\left(\begin{array}{cc}
2+\theta(t) & 0 \\
1 & 1.5
\end{array}\right) \quad \theta \in[-1,1] \\
& \mathfrak{B}(t)=\left\{\begin{array}{c}
B=\left(\begin{array}{ll}
2 & 0 \\
0 & 1
\end{array}\right) \\
B_{f}=\left(\begin{array}{ll}
2 & 0 \\
0 & 0
\end{array}\right)
\end{array} \quad t \geq t_{f} \quad C=\left(\begin{array}{ll}
1 & 0 \\
0 & 1
\end{array}\right)\right.
\end{aligned}
$$

and $\operatorname{sat}(u)$ as in (7)-(8) with $u_{j}^{M A X}=10, j=1,2$.

When the output-feedback controller designed in [22] is applied (referred in the following as controller 1), i.e. when the controller is designed without maximizing the tolerated fault isolation delay, the application of Theorem 2 gives a value $\lambda=1.9197$. On the other hand, if the controller is designed using Corollary 2 (referred in the following as controller 2), a value $\lambda=0$ is achieved. Notice that achieving the case $\lambda=0$, that would correspond to $\hat{t}_{I}=\infty$ using (44), is equivalent to the existence of a nominal controller that is robust against the considered fault. For the sake of additional comparison, let us also consider a controller designed using a prefixed value of $\lambda$, i.e. $\lambda=1$, which will be denoted as controller 3.

Let us consider a simulation that lasts $20 s$ with $x(0)=$ $\left(\begin{array}{ll}1 & 0\end{array}\right)^{T}, x_{c}(0)=\left(\begin{array}{ll}0 & 0\end{array}\right)^{T}, \theta(t)=\sin (5 t)$, and $t_{f}=0.5 s$. Since $\left(x(0)^{T}, x_{c}(0)^{T}\right) \in \mathcal{E}(P, 1)$ for all controllers 1,2 and 3 , the state trajectories will converge towards the origin and the control input will not saturate in the time interval $\left[0, t_{f}\right]$, as shown in Fig. 1. 


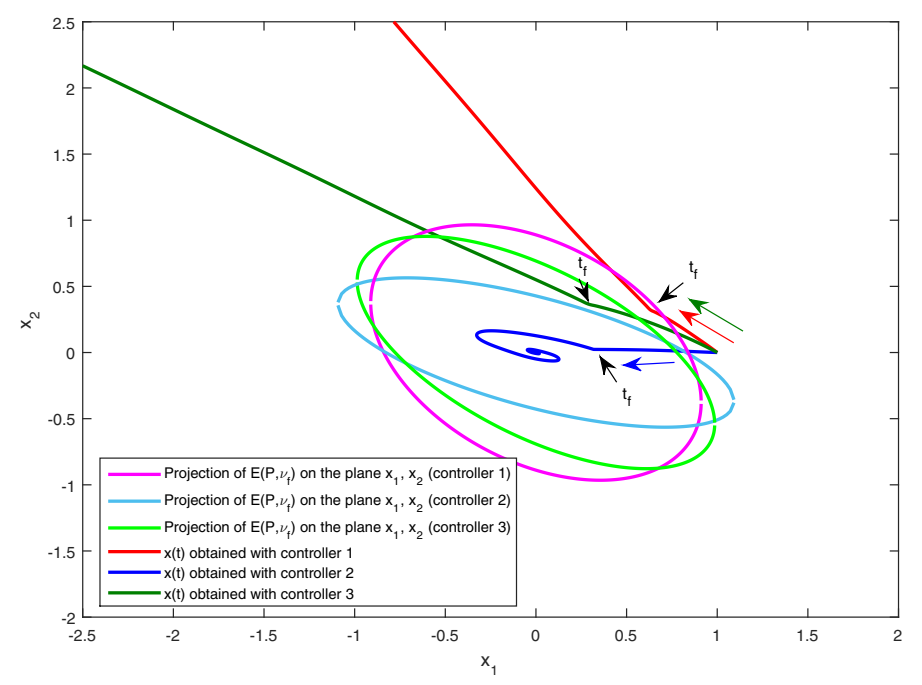

Fig. 1. State trajectories obtained with $t_{f}=0.5 \mathrm{~s}$ and no fault isolation during the simulation $\left(t_{I} \rightarrow \infty\right)$.

Assuming that the fault is not isolated during the simulation $\left(t_{I} \rightarrow \infty\right)$, it can be seen that the state trajectories obtained with controllers 1 and 3 diverge after the fault occurrence (see red and green lines in Fig. 1). On the other hand, the trajectory of the closed-loop system obtained with controller 2 keeps converging despite the fault occurrence and the absence of fault isolation (see blue line in Fig. 1). As a matter of fact, using Theorem 2, it can be calculated that for controller $1 \hat{t}_{I}=0.525 \mathrm{~s}$, while various simulations have shown that $\widehat{t}_{I}=0.748 \mathrm{~s}$. On the other hand, for controller 3 , $\hat{t}_{I}=0.698 \mathrm{~s}$ and $\widehat{t}_{I}=1.066 \mathrm{~s}$, which confirms that controller 1 is more sensitive to fault isolation delays than controller 3 , whereas controller 2, which has been obtained applying the proposed methodology, is tolerant w.r.t. any fault isolation delay.

The control inputs are shown in Fig. 2, where it can be seen that, due to the state trajectory divergence, the control inputs obtained with controllers 1 and 3 diverge as well. On the other hand, the control inputs obtained with controller 2 are within the saturation limits both before and after the fault occurrence.

Finally, Fig. 3 shows the state trajectories obtained for $t_{I}=3 \mathrm{~s}$. In this case, controller 1 is not able to recover the system's stability after the fault is isolated. On the other hand, the trajectory obtained with controller 3 converges asymptotically to the origin, although with a worse performance than the one obtained with controller 2.

\section{CONCLUSIONS AND FUTURE WORK}

This paper has presented a virtual actuator-based FTC strategy for LPV systems that are subject to saturation of the actuators and FDI delays. The developed design methodology allows maximizing the tolerated delay between the fault occurrence and its isolation. Under the assumption that the LPV system is polytopic, the design process consists in finding the optimal feasible solution to a finite set of

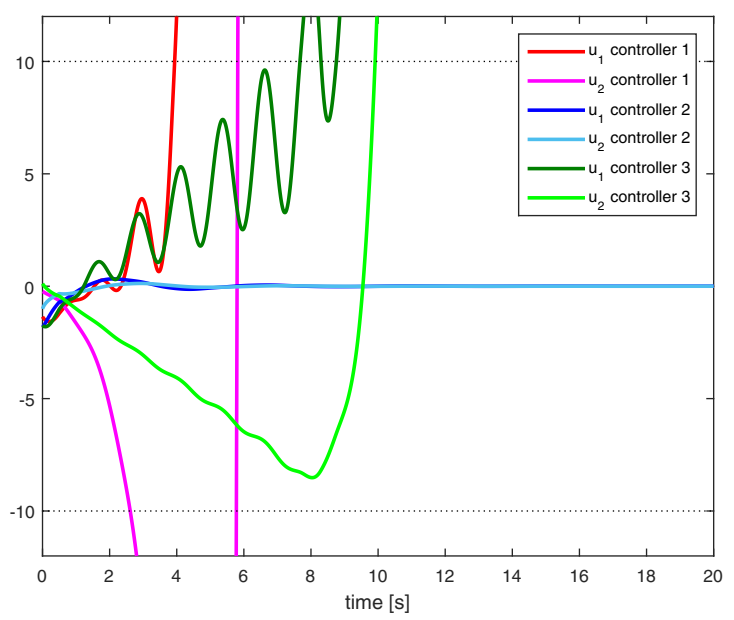

Fig. 2. Control inputs obtained with $t_{f}=0.5 \mathrm{~s}$ and no fault isolation during the simulation $\left(t_{I} \rightarrow \infty\right)$.

LMIs. The results achieved using an academic example have shown that the proposed design improves the performances with respect to a more traditional design that does not take into account the presence of FDI delays. In particular, it has been shown that, as a particular case, the proposed design methodology provides a nominal controller that is robust against the considered faults.

Future work will aim at improving the obtained results by considering: (i) the presence of sensor noise and process disturbances; (ii) parameter-varying input and output matrices; (iii) different indices, which will allow analyzing the trade-off between the tolerated fault isolation delay and the controller performance. 


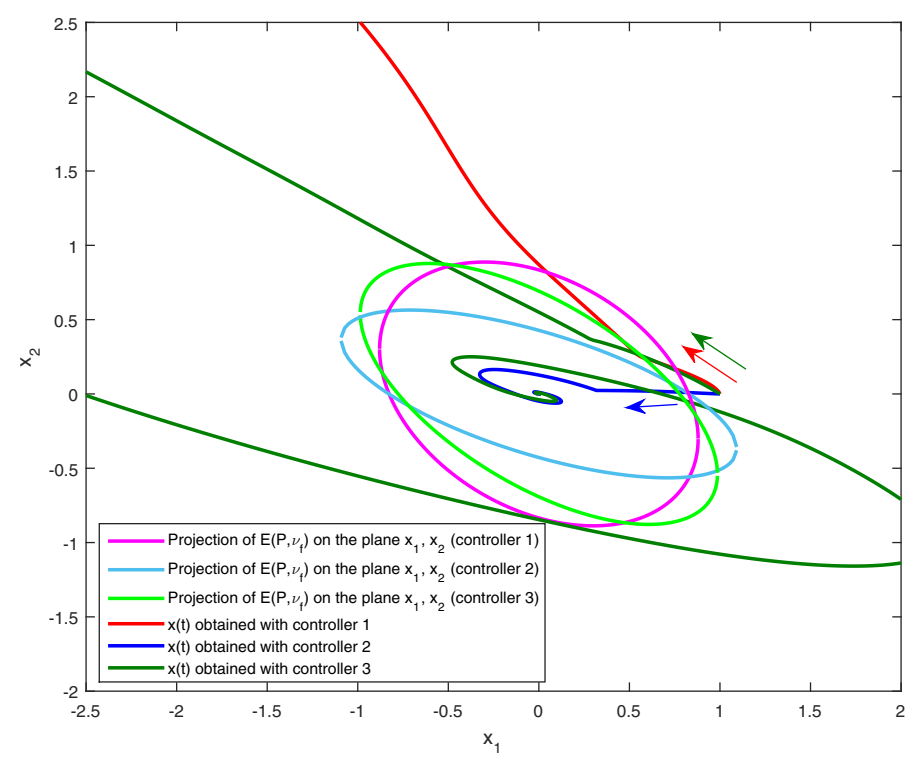

Fig. 3. State trajectories obtained with $t_{f}=0.5 \mathrm{~s}$ and $t_{I}=3 \mathrm{~s}$.

\section{REFERENCES}

[1] S. Tarbouriech and M. Turner, "Anti-windup design: an overview of some recent advances and open problems," IET Control Theory and Applications, vol. 3, no. 1, pp. 1-19, 2009.

[2] D. Dai, T. Hu, A. R. Teel, and L. Zaccarian, "Output feedback design for saturated linear plants using deadzone loops," Automatica, vol. 45, no. 12, pp. 2917-2924, 2009.

[3] X. Wu and Z. Lin, "Dynamic anti-windup design in anticipation of actuator saturation," International Journal of Robust and Nonlinear Control, vol. 24, no. 2, pp. 295-312, 2014.

[4] J. M. Gomes da Silva Jr., M. Z. Oliveira, D. Coutinho, and S. Tarbouriech, "Static anti-windup design for a class of nonlinear systems," International Journal of Robust and Nonlinear Control, vol. 24, pp. 793-810, 2014.

[5] S. Bezzaoucha, B. Marx, D. Maquin, and J. Ragot, "State and output feedback control for Takagi-Sugeno systems with saturated actuators," International Journal of Adaptive Control and Signal Processing, vol. Early View, 2015.

[6] H. Li, J. Wang, and P. Shi, "Output-feedback based sliding mode control for fuzzy systems with actuator saturation," accepted in IEEE Transactions on Fuzzy Systems, 2016.

[7] Q. Zhou, P. Shi, Y. Tian, and M. Wang, "Approximation-based tracking control for MIMO nonlinear systems with input saturation," IEEE Transactions on Cybernetics, vol. 45, no. 10, pp. 2119-2128, 2015.

[8] S. Tarbouriech, G. Garcia, J. M. Gomes da Silva Jr., and I. Queinnec, Stability and stabilization of linear systems with saturating actuators. Springer-Verlag London, 2011.

[9] L. Zaccarian and A. R. Teel, Modern anti-windup synthesis: control augmentation for actuator saturation. Princeton University Press, 2011.

[10] M. Blanke, M. Kinnaert, J. Lunze, and M. Staroswiecki, Diagnosis and Fault-Tolerant Control. Springer, 2006.

[11] H. Noura, D. Theilliol, J. C. Ponsart, and A. Chamseddine, Faulttolerant control systems: Design and practical applications. Springer London, 2009.

[12] J. Lan and R. Patton, "A new strategy for integration of fault estimation within fault-tolerant control," Automatica, vol. 69, pp. 48-59, 2016.

[13] A. Chamseddine, D. Theilliol, Y. M. Zhang, C. Join, and C. A. Rabbath, "Active fault-tolerant control system design with trajectory re-planning against actuator faults and saturation: Application to a quadrotor unmanned aerial vehicle," International Journal of Adaptive Control and Signal Processing, vol. 29, no. 1, pp. 1-23, 2015.

[14] Z. Wang, M. Rodrigues, D. Theilliol, and Y. Shen, "Fault-tolerant control for discrete linear systems with consideration of actuator saturation and performance degradation," in Proceedings of the 9th IFAC SAFEPROCESS, 2015, pp. 499-504.
[15] X. Qi, D. Theilliol, J. Qi, Y. Zhang, and J. Han, "Self-healing control against actuator stuck failures under constraints: application to unmanned helicopters," in Advanced and Intelligent Computations in Diagnosis and Control, Z. Kowalczuk, Ed. Springer, 2016.

[16] D. Rotondo, J.-C. Ponsart, D. Theilliol, F. Nejjari, and V. Puig, "A virtual actuator approach for the fault tolerant control of unstable linear systems subject to actuator saturation and fault isolation delay," Annual Reviews in Control, vol. 39, pp. 68-80, 2015.

[17] J. Lunze and T. Steffen, "Control reconfiguration after actuator failures using disturbance decoupling methods," IEEE Transactions on Automatic Control, vol. 51, pp. 1590-1601, 2006.

[18] D. Rotondo, F. Nejjari, and V. Puig, "A virtual actuator and sensor approach for fault tolerant control of LPV systems," Journal of Process Control, vol. 24, pp. 203-222, 2014.

[19] L. Dziekan, M. Witczak, and J. Korbicz, "Active fault-tolerant control design for Takagi-Sugeno fuzzy systems," Bulletin of the Polish Academy of Sciences, vol. 59, pp. 93-102, 2011.

[20] D. Rotondo, F. Nejjari, V. Puig, and J. Blesa, "Model reference FTC for LPV systems using virtual actuators and set-membership fault estimation," International Journal of Robust and Nonlinear Control, vol. 25 , no. 5 , pp. 735-760, 2015.

[21] M. M. Seron, J. A. De Doná, and J. Richter, "Fault tolerant control using virtual actuators and set-separation detection principles," International Journal of Robust and Nonlinear Control, vol. 22, no. 7, pp. 709-742, 2012.

[22] D. Rotondo, J.-C. Ponsart, D. Theilliol, F. Nejjari, and V. Puig, "Fault tolerant control of unstable LPV systems subject to actuator saturations using virtual actuators," in Proceedings of the 9th IFAC SAFEPROCESS, 2015, pp. 18-23.

[23] J. S. Shamma, "An overview of LPV systems," in Control of Linear Parameter Varying Systems with Applications, J. Mohammadpour and C. Scherer, Eds. Springer, 2012.

[24] T. Hu, Z. Lin, and B. M. Chen, "An analysis and design method for linear systems subject to actuator saturation and disturbance," Automatica, vol. 38, pp. 351-359, 2002.

[25] P. Apkarian, P. Gahinet, and G. Becker, "Self-scheduled $H_{\infty}$ Control of Linear Parameter-Varying Systems: A Design Example," Automatica, vol. 31 , no. 9 , pp. 1251 - 1261, 1995 .

[26] Z. Weng, R. Patton, and P. Cui, "Active fault-tolerant control for unstable systems," in Proceedings of the 4th IFAC Symposium on mechatronic systems, 2006, pp. 79-84.

[27] M. Chilali and P. Gahinet, " $H_{\infty}$ Design with Pole Placement Constraints: An LMI Approach," IEEE Transactions on Automatic Control, vol. 41, no. 3, pp. 358-367, 1996.

[28] R. A. Horn and C. R. Johnson, Matrix Analysis. Cambridge University Press, 1990. 